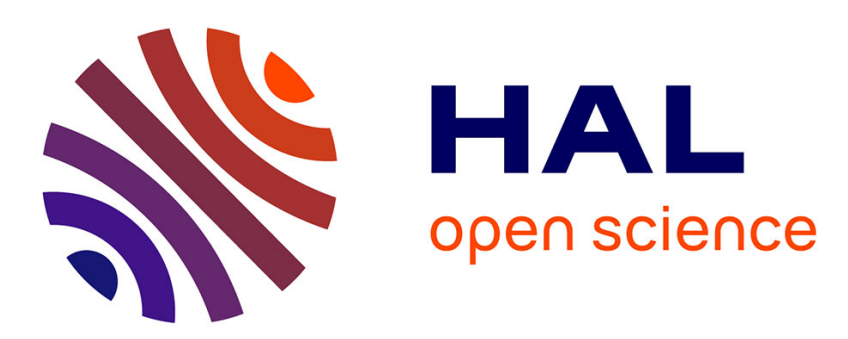

\title{
Lyapunov-based control of non isothermal continuous stirred tank reactors using irreversible thermodynamics.
}

Ha Hoang, Françoise Couenne, Christian Jallut, Yann Le Gorrec

\section{To cite this version:}

Ha Hoang, Françoise Couenne, Christian Jallut, Yann Le Gorrec. Lyapunov-based control of non isothermal continuous stirred tank reactors using irreversible thermodynamics.. Journal of Process Control, 2012, 22 (2), pp.412-422. 10.1016/j.procont.2011.12.007 . hal-00803656

\section{HAL Id: hal-00803656 https://hal.science/hal-00803656}

Submitted on 22 Mar 2013

HAL is a multi-disciplinary open access archive for the deposit and dissemination of scientific research documents, whether they are published or not. The documents may come from teaching and research institutions in France or abroad, or from public or private research centers.
L'archive ouverte pluridisciplinaire HAL, est destinée au dépôt et à la diffusion de documents scientifiques de niveau recherche, publiés ou non, émanant des établissements d'enseignement et de recherche français ou étrangers, des laboratoires publics ou privés. 


\title{
Lyapunov-based control of non isothermal continuous stirred tank reactors using irreversible thermodynamics
}

\author{
H. Hoang, F. Couenne, C. Jallut, and Y. Le Gorrec,
}

\begin{abstract}
In this paper, the thermodynamic availability function is used as a Lyapunov function for the practical derivation of non linear control laws for the stabilization of a large class of CSTRs far from the equilibrium. The strict convexity of the availability function is guaranteed as long as one of the extensive variables is fixed. In this study, we consider liquid mixture with constant volume, the constraint on the volume being insured by perfect regulation of the outlet flow of the CSTR. Several control laws are then derived which insure global asymptotic stability, exponential stability or simple asymptotic stability. These control laws are discussed regarding the magnitude and the dynamic variations of the control variable. It is shown that the availability function can be split into two parts : one corresponds to the mixing term and depends on mole numbers only and the other depends on both temperature and mole numbers. The two parts are positive and the second one is chosen as a new Lyapunov function. The use of this new Lyapunov function insures smooth variations of the control variable. An exothermal, first order chemical reaction leading to multiple steady-state operating points of the CSTR illustrates the proposed theory.
\end{abstract}

\section{Index Terms}

Lyapunov based control, thermodynamics, non isothermal CSTR.

H. Hoang, F. Couenne and C. Jallut are with the LAGEP, UMR CNRS 5007, University of Lyon1, Villeurbanne, France, (e-mails: \{hoang;couenne;jallut\}@lagep.univ-lyon1.fr).

Y. Le Gorrec is with FEMTO-ST / AS2M ENSMM Besançon, Besançon, France (e-mail: yann.le.gorrec@ens2m.fr). 


\section{INTRODUCTION}

Stability analysis and control design are usually achieved by using energetic considerations, as in the Lyapunov theory [29] or more generally in approaches based on passivity [41]. Such tools are very efficient when electrical or mechanical systems are considered as the links between energy and system dynamics are well established. The port Hamiltonian framework is commonly used [8, 37, 38] for mechanical or electrical systems and the energy function traditionally used in theses cases is restricted to that of the considered domain (electrical or mechanical). As a consequence, the energy is usually not conserved. Dissipation corresponds to the irreversible transfer of electrical or mechanical energy to the thermal domain that is not explicitly represented.

In thermodynamic systems, especially in the case of reaction processes, the total energy of the system is represented by the internal energy. Internal energy refers to the energy of matter at an atomic and molecular level. From the first law of thermodynamics it is a conserved quantity. As a consequence, the internal energy cannot be used as a storage function; indeed dissipation due to reaction irreversibly transfers energy from the material domain to the thermal domain, these two domains being represented within the internal energy. So one has to clarify the links existing between thermodynamics and system dynamics to exhibit a Lyapunov function candidate able to capture the irreversibility of the system for stability analysis and control design. Such studies initiated by the Brussels school of thermodynamics [18] led to active research activities in the process control domain. Indeed recently numerous researchers [1, 2, 16, 20, 42] proposed an insightful study of the thermodynamic availability function as defined previously in $[9,30]$ and its use for analysis and control design for some classes of thermodynamic systems. At the same time numerous works have been devoted to the modeling of irreversible thermodynamic systems using the port Hamiltonian framework [13, 15, 21, 32, 25]. Even if such formulation does not formally exist [14], a pseudo Hamiltonian formulation has proved to help to find the appropriate control law for a class of simple chemical reactors for example [15, 32].

This paper is concerned with the non linear control of Continuous Stirred Tank reactors (CSTRs). Such systems represent numerous difficulties that system control theory has to overcome : they are non linear, they may have multiple steady states and coupling between energy and material balances. For all theses reasons, CSTRs have been widely studied in literature with respect to control design, by using classical approaches (see for example $[3,5,31,7,17,19$, 
$26,27,31,33,34,39,40])$ or more recently by using thermodynamic based approaches through the so called availability function [20, 23, 28, 35, 42] or Hamiltonian framework [13, 15, 21$]$. Nevertheless a majority of the thermodynamics based approaches consider strong assumptions about the constitutive equations (diffusion laws, reaction rate) to use passivity properties for control purpose or to exhibit system structural properties. Furthermore they are mainly devoted to infinite dimensional thermodynamic systems, process network or inventory control. It appears that even for simple reactions, control issues using thermodynamic properties are still open problems. The case of non isothermal CSTRs control by using thermodynamics was addressed in [4]. The authors proposed a hierarchical control formed by inventory controllers for the extensive variables combined with "classical" controllers such as PI controller for the intensive variables. The authors do not use the availability function for the control design. They verify a posteriori that the controller devoted to the intensive variables modifies the entropy production. Furthermore they do not use the thermodynamic chemical potential for the control but the concentrations. In these control strategies the enthalpy of reaction and the heat capacities are considered as constant.

The objective of the current paper is twofold. First to derive stabilizing control laws directly from the thermodynamic availability function for a general class of CSTRs with time varying enthalpies of reaction and heat capacities. To remain in the thermodynamic context we have to use intensive variables such as chemical potentials. Moreover, the use of the availability function needs the use of a controller to fix at least one of the extensive variables of the system. In this paper we consider that there exists a "perfect" CSTR outlet flow control to keep the volume constant.

Second, it will be shown that the availability function is very conservative with respect to the magnitude and the dynamics of the control variables. To overcome this drawback, we modify the initial Lyapunov function by excluding a non-thermal part that is nonlinear with respect to the mole numbers.

This paper is organized as follows. In Section 2, thermodynamic properties of homogeneous mixtures are described. We recall how the availability function is defined in [42] and how it is used as a Lyapunov function. Section 3 is devoted to the non-linear control of CSTRs. Three control strategies are presented and analyzed with respect to the closed loop stability and actuators limitations. Section 4 provides an illustrative example. Section 5 ends the paper with concluding 
remarks and perspectives.

\section{IRREVERSIBLE THERMODYNAMICS AND STABILITY}

In this section we review the main thermodynamic concepts necessary for the stability analysis of CSTRs. As stated in the introduction, the internal energy cannot be used for control purposes, as it is a conserved quantity. One has to investigate the notion of entropy and study the associated potential functions usable for closed loop stabilization. We restrict our study to the case of homogeneous mixtures at constant pressure. Discussions related to phase separation and multiphasic mixtures can be found in [28].

\section{A. Overview of thermodynamic concepts}

In equilibrium thermodynamics, the system variables are divided into extensive and intensive variables, depending on whether their values depend on the "size" of the system (extensive variables) or not (intensive variables). One can cite as an example of extensive variables the internal energy $U$, the volume $V$, the entropy $S$ or the mole number $n_{i}$ for each species $i$ $\left(i=1, \cdots, n_{c}\right)$ of the homogeneous mixture under consideration.

The intensive variables are the variables that are energy conjugate to the intensive variable: their product has the dimension of an energy. The internal energy of a homogeneous system is then expressed in terms of products of pairings of energy conjugate variables such as pressure $P$ - volume $V$, temperature $T$ - entropy $S$ and chemical potential $\mu_{i}$ - mole number $n_{i}$. The variation of the internal energy is derived from the variation of the extensive variables using the Gibbs equation [36]:

$$
d U=T d S-P d V+\sum_{i=1}^{n_{c}} \mu_{i} d n_{i}
$$

Equivalently one can write the variation of the entropy as:

$$
d S=\frac{1}{T} d U+\frac{P}{T} d V+\sum_{i=1}^{n_{c}} \frac{-\mu_{i}}{T} d n_{i} .
$$

or in vectorial form:

$$
d S=\mathbf{w}_{\mathbf{U}}^{T} d \mathbf{Z}_{\mathbf{U}}
$$

with $\mathbf{w}_{\mathbf{U}}^{T}=\left(\frac{1}{T}, \frac{P}{T}, \frac{-\mu_{1}}{T}, \cdots, \frac{-\mu_{n_{c}}}{T}\right)$ and $\mathbf{Z}_{\mathbf{U}}^{T}=\left(U, V, n_{1}, \cdots, n_{n_{c}}\right)$. When isobaric conditions are considered, it is more convenient to use the enthalpy $H$ instead of the internal 
energy $U$. Indeed $H\left(S, P, n_{i}\right)$ is obtained as the Legendre transform of $U\left(S, V, n_{i}\right)$ with respect to the volume $V$. Using the fact that $H=U+P V$, one can write:

$$
d H=d U+d(P V)=T d S+V d P+\sum_{i=1}^{n_{c}} \mu_{i} d n_{i}
$$

which reduces to:

$$
d H=d U+d(P V)=T d S+\sum_{i=1}^{n_{c}} \mu_{i} d n_{i}
$$

when the pressure is assumed to be constant. As a consequence in this case:

$$
d S=\frac{1}{T} d H+\sum_{i=1}^{n_{c}} \frac{-\mu_{i}}{T} d n_{i}
$$

which can be written as:

$$
d S=\mathbf{w}^{T} d \mathbf{Z}
$$

with $\mathbf{w}^{T}=\left(\frac{1}{T}, \frac{-\mu_{1}}{T}, \cdots, \frac{-\mu_{n_{c}}}{T}\right)$ and $\mathbf{Z}^{T}=\left(H, n_{1}, \cdots, n_{n_{c}}\right)$.

Since the entropy $S$ is an extensive variable, it is a homogenous function of degree 1 of $\mathbf{Z}$ $(\text { see [9] })^{1}$. So from Euler's theorem ${ }^{2}[9,36]$, we obtain:

$$
S(Z)=\mathbf{w}^{T} \mathbf{Z}
$$

Equation (7) or (3) can also be applied in irreversible thermodynamics as soon as the local equilibrium assumption is assumed: it postulates that the present state of the homogeneous system in any evolution can be characterized by the same variables like those at equilibrium and is independent of the rate of evolution [18].

As a consequence of (7) or (3), the time derivative of the entropy can alternatively be written as:

$$
\frac{d S}{d t}=\mathbf{w}^{T} \frac{d \mathbf{Z}}{d t}
$$

Based on the second law of thermodynamics in the case of homogeneous thermodynamical systems, the entropy function $S(Z)$ is concave with respect to its arguments $\mathbf{Z}$ (see [9]). Indeed from equation (7) and Euler's equation (8), the intersection of the map $S$ with its tangent plane

\footnotetext{
${ }^{1}$ Let $f: \mathbb{R}_{+}^{n} \rightarrow \mathbb{R}$. The function $f$ is homogeneous of degree $k$ if for all $x \in \mathbb{R}_{+}^{n}$ and $\alpha>0, f(\alpha x)=\alpha^{k} f(x)$.

${ }^{2}$ If $f: \mathbb{R}_{+}^{n} \rightarrow \mathbb{R}$ is continuously differentiable and homogeneous of degree $k>0$, then $\sum_{i=1}^{n} \frac{\partial f}{\partial x_{i}} x_{i}=k f\left(x_{1}, \cdots, x_{n}\right)$
} 
in $S(\mathbf{Z})$ is a straight line of slope $\mathbf{w}^{T}$. Intensive variables $\mathbf{w}^{T}$ are homogeneous functions of degree 0 of $\mathbf{Z}$. This implies that all the pairs of points of the state space, $\mathbf{Z}_{\alpha}$ and $\mathbf{Z}_{\beta}$ such that:

$$
\frac{H_{\alpha}}{H_{\beta}}=\frac{n_{i \alpha}}{n_{i \beta}}=\lambda, \quad i=1, \cdots, n_{c} ; \lambda \in \mathbb{R}
$$

have the same slope $\mathbf{w}_{\alpha}=\mathbf{w}_{\beta}$. As a consequence $S$ is not strictly concave but admits a tangent hypersurface defined by (10). $\lambda$ is fixed as soon as one of the extensive variables such as $n_{k}$, the total mole number $N=\sum_{k} n_{k}, V=\sum_{k} n_{k} v_{k}, H=\sum_{k} n_{k} h_{k}$, or the total mass $M=\sum_{k} n_{k} m_{k}$ is fixed. In these expressions $v_{k}, h_{k}$ and $m_{k}$ are the partial molar volume, partial molar enthalpy and molar mass of the component $k$. In this case the entropy function $S$ becomes strictly concave.

Strict concavity of $S$ with respect to some extensive variable $\mathbf{Z}$ induces that the intersection between the tangent line and $S(\mathbf{Z})$ is a point. Such a situation is depicted in Fig. 1.

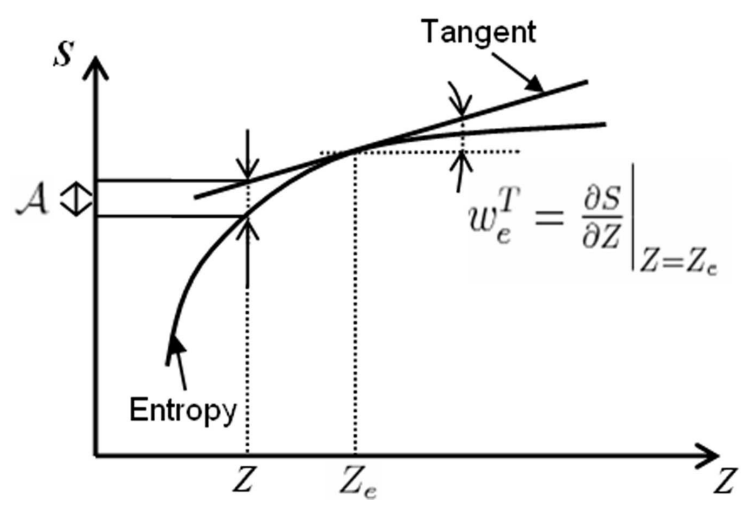

Fig. 1. Entropy and availability functions with respect to $\mathbf{Z}$.

From these observations, it can be shown (see [42]) that the non negative function:

$$
\mathcal{A}(\mathbf{Z})=S_{e}+\mathbf{w}_{\mathbf{e}}^{T}\left(\mathbf{Z}-\mathbf{Z}_{\mathbf{e}}\right)-S(\mathbf{Z})
$$

where $\mathbf{Z}_{\mathrm{e}}$ is a fixed reference state (as an example the desired set point for closed loop control) satisfying the aforementioned constraint, is such that:

$$
\mathcal{A}(\mathbf{Z})>0 \quad \forall \mathbf{Z} \neq \mathbf{Z}_{\mathbf{e}}, \quad \mathcal{A}\left(\mathbf{Z}_{\mathbf{e}}\right)=0
$$

$\mathcal{A}(\mathbf{Z})$ is the algebraic distance between the entropy $S(\mathbf{Z})$ and the tangent plane passing through $\mathbf{Z}_{\mathbf{e}}$. This function is called availability function and is in this case strictly convex and usable as 
a Lyapunov function for closed loop control purpose. Let us consider Example 1 to illustrate the fact that the availability function is strictly convex as soon as one extensive variable is fixed.

Example 1 (Strict convexity of the Availability function: illustrative example): We consider the mixing entropy of an ideal solution composed of two species as given by:

$$
\Delta S^{m}\left(n_{1}, n_{2}\right)=-R n_{1} \ln \left(\frac{n_{1}}{n_{1}+n_{2}}\right)-R n_{2} \ln \left(\frac{n_{2}}{n_{1}+n_{2}}\right)
$$

One can easily check that $\Delta S^{m}\left(n_{1}, n_{2}\right)$ is a first order homogeneous function concave with respect to $n_{1}$ and $n_{2}$ and

$$
\left(\frac{\partial \Delta S^{m}}{\partial n_{1}}, \frac{\partial \Delta S^{m}}{\partial n_{2}}\right)=\left(-R \ln \left(\frac{n_{1}}{n_{1}+n_{2}}\right),-R \ln \left(\frac{n_{2}}{n_{1}+n_{2}}\right)\right)
$$

are zero order homogeneous functions with respect to $n_{1}$ and $n_{2}$. In this case $\mathcal{A}$ is defined by:

$$
\begin{aligned}
\mathcal{A}\left(n_{1}, n_{2}\right) & =R n_{1}\left(\ln \left(\frac{n_{1}}{n_{1}+n_{2}}\right)-\ln \left(\frac{n_{1 e}}{n_{1 e}+n_{2 e}}\right)\right) \\
& +R n_{2}\left(\ln \left(\frac{n_{2}}{n_{1}+n_{2}}\right)-\ln \left(\frac{n_{2 e}}{n_{1 e}+n_{2 e}}\right)\right)
\end{aligned}
$$

One can check that $\mathcal{A}\left(n_{1 e}, n_{2 e}\right)=\mathcal{A}\left(\lambda n_{1 e}, \lambda n_{2 e}\right)=0, \forall \lambda \in \mathbb{R}$ and $\mathcal{A}$ is not strictly convex. This situation is represented in Fig. 2 by the contact line. If one imposes $N=n_{1}+n_{2}$ constant then $\mathcal{A}$ becomes strictly convex and can be considered as a Lyapunov function candidate. This last situation is depicted in Fig. 1.

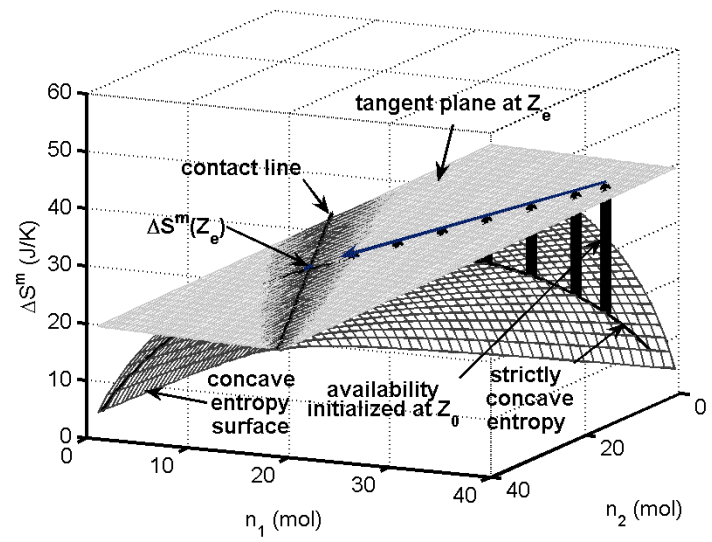

Fig. 2. $\Delta S$ function and its tangent plane. 
It is important to note that the positivity of the availability function comes from thermodynamic properties of the homogeneous mixture. Yet the dynamic behavior of the system only depends on the balance equations.

\section{B. Thermodynamic properties and stabilization}

In the previous subsection we have shown that from thermodynamic properties of the entropy function one can exhibit a positive function that is strictly convex and that vanishes in $\mathbf{Z}_{\mathbf{e}}$. In the following we focus our attention on the dynamic control of CSTRs. We will show that these thermodynamic systems can be described by a set of ordinary differential equations of the form:

$$
\frac{d \mathbf{Z}}{d t}=f(\mathbf{Z})+g(\mathbf{Z}) u
$$

The idea is to use the availability function as Lyapunov function and to find an appropriate control strategy so that this function decreases with time. This problem can be formulated as follows.

Control Problem. The stabilization of the thermodynamic system (17) about the desired equilibrium point $\mathbf{Z}_{\mathbf{e}}$ is the problem of designing a feedback control law $u(\mathbf{Z})$ in (17) so that:

$$
\begin{array}{r}
\frac{d \mathcal{A}}{d t}=\tilde{\boldsymbol{w}}^{T} \frac{d \mathbf{Z}}{d t}<0 \text { where } \tilde{\boldsymbol{w}}=\left(\begin{array}{c}
\tilde{w}_{1} \\
\tilde{\boldsymbol{w}}_{\mathbf{2}}
\end{array}\right) \\
\text { with } \tilde{w}_{1}=\frac{1}{T}-\frac{1}{T_{e}}, \quad \tilde{\boldsymbol{w}}_{\mathbf{2}}=\frac{-\boldsymbol{\mu}}{T}+\frac{\boldsymbol{\mu}_{e}}{T_{e}}
\end{array}
$$

\section{THERMODYNAMIC MODELING AND NONLINEAR CONTROL OF CSTRS}

\section{A. General modeling of CSTRs}

We first recall the considered assumptions.

Assumptions 1. We consider a liquid homogeneous mixture of $n_{c}$ species having the following properties:

1. The reacting mixture is assumed to be ideal and incompressible.

2. Isobaric conditions are considered. 
3. The total volume $V$ is assumed to be constant. This constraint is satisfied by perfect output molar flow regulation.

4. The CSTR is controlled through the jacket heat flow $\dot{Q}$.

Assumption 1.2 allows $H$ instead of $U$ to be considered for the modelling. Hypothesis 1.3 guarantees the strict concavity of the entropy function (in the following one could have considered constraint on the total mass number without significant change or computational difficulty) and consequently the strict positivity of the availability function $[1,2]$, i.e.:

$$
\mathcal{A}(\mathbf{Z})=-\left(\mathbf{w}-\mathbf{w}_{\mathbf{e}}\right)^{T} \mathbf{Z}>0, \forall \mathbf{Z} \neq \mathbf{Z}_{\mathbf{e}}, \mathcal{A}\left(\mathbf{Z}_{\mathbf{e}}\right)=0 \text { for } \mathbf{Z}=\mathbf{Z}_{\mathbf{e}}
$$

The species balances within the CSTR can be written:

$$
\frac{d \mathbf{n}}{d t}=\mathbf{F}_{\mathbf{i}}-\mathbf{F}_{\mathbf{o}}+V \boldsymbol{\nu}^{T} \mathbf{r}
$$

where $\mathbf{n}$ is the vector of mole numbers, $\mathbf{F}_{\mathbf{i}}, \mathbf{F}_{\mathbf{o}}$ the inlet and outlet molar flows respectively, $\boldsymbol{\nu}$ the matrix of stoichiometric coefficients and $\mathbf{r}$ the vector of reaction rates. Let us now consider the energy balance equation involving the enthalpy variable $H$ in the case of the isobaric condition [36]:

$$
\frac{d H}{d t}=\mathbf{h}_{\mathbf{i}}^{T} \mathbf{F}_{\mathbf{i}}-\mathbf{h}_{\mathbf{o}}^{T} \mathbf{F}_{\mathbf{o}}+\dot{Q}
$$

where $\mathbf{h}_{\mathbf{i}}, \mathbf{h}_{\mathbf{o}}$ are the vectors of partial molar enthalpies respectively at the inlet and the outlet of the CSTR, $\dot{Q}$ is the heat flow exchanged with the jacket.

By expressing the outlet molar flows as a function of inlet molar flows and mole numbers the volume of the mixture is defined by:

$$
V=\mathbf{v}^{T} \mathbf{n}
$$

where $\mathbf{v}$ is the vector of molar partial volumes which are assumed to be constant. As a consequence the constraint on the total volume can be written:

$$
\frac{d V}{d t}=\mathbf{v}^{T} \frac{d \mathbf{n}}{d t}=0 \text { or equivalently } \mathbf{v}^{T} \mathbf{F}_{\mathbf{i}}-\mathbf{v}^{T} \mathbf{F}_{\mathbf{o}}+V \mathbf{v}^{T} \boldsymbol{\nu}^{T} \mathbf{r}=0
$$

$\mathbf{F}_{\mathbf{o}}$ can be expressed with respect to the total molar flow rate $F$ as:

$$
\mathbf{F}_{\mathbf{o}}=\frac{\mathbf{n}}{N} F \text { then } \mathbf{v}^{T} \mathbf{F}_{\mathbf{o}}=\frac{V}{N} F
$$

where $N=\sum_{k} n_{k}$ is the total number of moles. From (24) we obtain:

$$
F=\frac{N \mathbf{v}^{T}}{V}\left(\mathbf{F}_{\mathbf{i}}+V \boldsymbol{\nu}^{T} \mathbf{r}\right) \text { and } \mathbf{F}_{\mathbf{o}}=\frac{\mathbf{n} \mathbf{v}^{T}}{V}\left(\mathbf{F}_{\mathbf{i}}+V \boldsymbol{\nu}^{T} \mathbf{r}\right)
$$


Consequently the constraint on the volume is guaranteed as soon as the outlet molar flow is chosen equal to (26). To summarize the balances on extensive variables can be written:

$$
\frac{\mathbf{d Z}}{\mathbf{d t}}=\mathbf{f}(\mathbf{Z})+\mathbf{g}(\mathbf{Z}) u
$$

with:

$$
\left\{\begin{array}{l}
\mathbf{f}=\left(\begin{array}{c}
f_{H} \\
\mathbf{f}_{\mathbf{n}}
\end{array}\right)=\left(\begin{array}{c}
\left(\mathbf{h}_{\mathbf{i}}^{T}-\mathbf{h}_{\mathbf{o}}{ }^{T} \frac{\mathbf{n v}^{T}}{V}\right) \mathbf{F}_{\mathbf{i}}-\mathbf{h}_{\mathbf{o}}{ }^{T} \mathbf{n} \mathbf{v}^{T} \boldsymbol{\nu}^{T} \mathbf{r} \\
\left(I_{n_{c}, n_{c}}-\frac{\mathbf{n v}^{T}}{V}\right)\left(V \boldsymbol{\nu}^{T} \mathbf{r}+\mathbf{F}_{\mathbf{i}}\right)
\end{array}\right) \\
\mathbf{g}=\left(\begin{array}{c}
1 \\
\mathbf{0}
\end{array}\right), u=\dot{Q}
\end{array}\right.
$$

From now on the constraint on the volume is implicitly taken into account in (27).

To end we consider the additional following assumptions [5]:

\section{Assumptions 2.}

1. At isothermal condition, $T=T_{e}$ corresponds a unique set of stationary mole numbers $\mathbf{n}_{\mathbf{e}}$.

2. The reaction kinetics are smooth and thus open loop dynamics $f_{H}$ and $\mathbf{f}_{\mathbf{n}}$ are smooth.

\section{B. Non linear control using $\mathcal{A}$ as a Lyapunov function}

In this subsection the heat flow $\dot{Q}$ is used as the control variable through state feedback i.e. $u=u(\mathbf{Z})$, to solve the Control Problem of subsection II-A. We are also interested in the applicability of the proposed control strategy. In particular the proposed control variable $u(\mathbf{Z})$ has to be bounded. It is not always true when global asymptotic stability is considered and consequently only simple asymptotic stability has to be considered. These considerations will lead to the modification of the Lyapunov function (Subsection III-C).

Let us first consider the global stabilization problem. The availability function $\mathcal{A}$ defined by (11) can be explicitly written:

$$
\mathcal{A}(\mathbf{Z})=-\tilde{\boldsymbol{w}}^{T} \mathbf{Z}=-\left(\frac{1}{T}-\frac{1}{T_{e}}\right) H-\left(\frac{-\boldsymbol{\mu}}{T}+\frac{\boldsymbol{\mu}_{e}}{T_{e}}\right)^{T} \mathbf{n}
$$

and from (27):

$$
\frac{d \mathcal{A}(\mathbf{Z})}{d t}=-\tilde{\boldsymbol{w}}^{T} \frac{d \mathbf{Z}}{d t}=-\tilde{w}_{1}^{T} f_{H}-\tilde{\boldsymbol{w}}_{2}^{T} \mathbf{f}_{\mathbf{n}}-\tilde{w}_{1}^{T} u
$$

The realization of the control law able to solve the aforementioned Control problem is obtained from (30) by inverting $\tilde{w}_{1}$ as stated in Proposition 1.

Proposition 1: The dynamic system $(27,28)$ is: 
- globally asymptotically stable in $\mathbf{Z}_{\mathrm{e}}$ if :

$$
u(\mathbf{Z})=-f_{H} \quad+\tilde{w}_{1}^{-1}\left(\left(\mathbf{Z}-\mathbf{Z}_{\mathbf{e}}\right)^{T} K(\mathbf{Z})\left(\mathbf{Z}-\mathbf{Z}_{\mathbf{e}}\right)-\tilde{\boldsymbol{w}}_{\mathbf{2}}^{\mathbf{T}} \mathbf{f}_{\mathbf{n}}\right)
$$

$K(Z)$ being a positive definite matrix valued function and $f_{H}, \mathbf{f}_{\mathrm{n}}$ defined by (28). In this case

$$
\frac{d \mathcal{A}}{d t}=-\left(\mathbf{Z}-\mathbf{Z}_{\mathbf{e}}\right)^{T} \mathbf{K}(\mathbf{Z})\left(\mathbf{Z}-\mathbf{Z}_{\mathbf{e}}\right)<0, \quad \forall \mathbf{Z} \neq \mathbf{Z}_{\mathbf{e}}
$$

- exponentially stable in $\mathbf{Z}_{\mathrm{e}}$ if:

$$
u(Z)=-f_{H}+\tilde{w}_{1}^{-1}\left(-\tilde{\boldsymbol{w}}_{2}^{T} \mathbf{f}_{\mathbf{n}}+K(\mathbf{Z}) \mathcal{A}\right)
$$

with $K(Z)$ a positive definite valued function and $\mathcal{A}$ defined from (11). In this case

$$
\frac{d \mathcal{A}}{d t}=-K(\mathbf{Z}) \mathcal{A}
$$

Proof. $\mathcal{A}(\mathbf{Z})$ is chosen as a Lyapunov function candidate. Let us now consider (30). Choosing $u(Z)$ defined by (31) guarantees that (32) is satisfied since $K(\mathbf{Z})$ is a positive matrix. $\mathcal{A}$ is then positive, equal to zero for $\mathbf{Z}=\mathbf{Z}_{\mathrm{e}}$. Its time derivative remains negative for all $\mathbf{Z} \neq \mathbf{Z}_{\mathrm{e}}$. Furthermore $\frac{d \mathcal{A}}{d t}$ tends to $-\infty$ if $\|u(\mathbf{Z})\|$ tends to $-\infty$. When (33) is considered $\mathcal{A}$ can be written

$$
\mathcal{A}=\mathcal{A}_{t=0} e^{-K t}
$$

Furthermore it is possible to write $\mathcal{A}$ on the form (see the proof of Lemma 1 for details):

$$
\mathcal{A}=-\left(1-\frac{T}{T_{e}}+\ln \left(\frac{T}{T_{e}}\right)\right) C_{p}-\sum_{k} n_{k} R \ln \left(\frac{n_{k e} \sum_{j} n_{j}}{n_{k} \sum_{j} n_{j e}}\right)
$$

where $C_{p}=\sum_{i} n_{i} c_{p i}$. From the constraint on the total volume the mole numbers are positive and bounded and then $0<C_{p \min } \leq C_{p} \leq C_{p \max }$. Furthermore in this case $-\sum_{k} n_{k} R \ln \left(\frac{n_{k e} \sum_{j} n_{j}}{n_{k} \sum_{j} n_{j e}}\right)$ is a positive bounded function. As a consequence there exist two positive constants $\kappa_{1}, \kappa_{2}$ such that:

$$
\kappa_{1}\left|T-T_{e}\right|<\mathcal{A}<\kappa_{2}\left|T-T_{e}\right|
$$

Equations (35) and (37) imply that $T$ exponentially converges to $T_{e}$. From Assumption 2.2 one can state that $n_{k}$ exponentially converge to $n_{k e}$ and then $H$ converges to $H_{e}$.

One problem is that the control law can be unbounded from the inversion of $\left(\frac{1}{T}-\frac{1}{T_{e}}\right)=\tilde{w}_{1}$. To avoid such undesirable behavior we can bound the Lyapunov function time derivative by a negative quadratic function of the intensive variable $\tilde{w}_{1}$ instead of the extensive variables $Z$, as 
stated in Proposition 2. Indeed, in this case, the control variable depends only on $\tilde{w}_{1}^{-1} \tilde{\boldsymbol{w}}_{2}^{T} \mathbf{f}_{\mathbf{n}}$ and this term remains bounded. Such property is difficult to prove in the general case. Nevertheless, this preliminary result will lead to the definition of a new Lyapunov function usable for control purpose. In this last case the boundedness of the control variable can be proved (cf. Theorem 1).

Proposition 2: The dynamic system defined by $(27,28)$ is asymptotically stable in $Z_{e}$ if :

$$
u(Z)=-f_{H}+K_{1}(\mathbf{Z}) \tilde{w}_{1}-\tilde{w}_{1}^{-1} \tilde{\boldsymbol{w}}_{\mathbf{2}}^{\boldsymbol{T}} \mathbf{f}_{\mathbf{n}}
$$

where $f_{H}$ and $\mathbf{f}_{\mathbf{n}}$ are defined by (28) and $K_{1}$ is a real positive constant ${ }^{3}$. In this case

$$
\frac{d \mathcal{A}}{d t}=-K_{1}(\mathbf{Z}) \tilde{w}_{1}^{2}<0 \quad \forall T \neq T_{e}
$$

and

$$
\lim _{\mathbf{Z} \rightarrow \mathbf{Z}_{\mathbf{e}}} u(\mathbf{Z})=0
$$

Proof. The inequality (39) is derived from equations (30) and (38). The time derivative of $\mathcal{A}(\mathbf{Z})$ remains negative for all $T \neq T_{e}$. From Assumption 2.1, the invariant set associated to $\dot{\mathcal{A}}(\mathbf{Z})=0$ at $T=T_{e}$ reduces to $\mathbf{Z}_{\mathbf{e}}=\left(H_{e}, \mathbf{n}_{\mathbf{e}}\right)$. Then from LaSalle's Theorem [29] the system trajectories converge asymptotically to the equilibrium state $\mathbf{Z}_{\mathbf{e}}$ and then $\mathbf{f}_{\mathbf{n}} \rightarrow 0, \frac{1}{T} \rightarrow \frac{1}{T_{e}}$ and $\frac{d T}{d t} \rightarrow 0$. Since the pressure influence is neglected, the variation of the molar enthalpy of a species $i$ is given by:

$$
d h_{i}=c_{p i} d T
$$

Then the time derivative of the enthalpy $H=\mathbf{n}^{T} \mathbf{h}$ can be written:

$$
\frac{d H}{d t}=C_{p} \frac{d T}{d t}+\mathbf{h}^{T} \frac{d \mathbf{n}}{d t}
$$

Let us now consider system (27) in closed loop with (38), i.e.:

$$
\frac{d H}{d t}=C_{p} \frac{d T}{d t}+\mathbf{h}^{T} \frac{d \mathbf{n}}{d t}=f_{H}+u
$$

with

$$
u(Z)=-f_{H}+K_{1} \tilde{w}_{1}-\left(\tilde{w}_{1}\right)^{-1} \tilde{\boldsymbol{w}}_{\mathbf{2}}^{\boldsymbol{T}} \mathbf{f}_{\mathbf{n}}
$$

\footnotetext{
${ }^{3}$ The proof remains true with $K_{1}(\mathbf{Z})$ a real valued positive function
} 
Then by using the notation $\mathbf{f}_{\mathbf{n}}=\frac{d \mathbf{n}}{d t}$ one can write:

$$
C_{p} \frac{d T}{d t}=K_{1} \tilde{w}_{1}-L(T, \mathbf{n})
$$

with $\left(\mathbf{c}_{\mathbf{p}}\right.$ being the vector of the partial heat capacities $\left.c_{p i}\right)$ :

$$
\left\{\begin{array}{l}
C_{p}=\mathbf{c}_{\mathbf{p}}{ }^{T} \mathbf{n}, \\
L(T, \mathbf{n})=\left(\tilde{w}_{1}^{-1} \tilde{\boldsymbol{w}}_{\mathbf{2}}^{\boldsymbol{T}}+\mathbf{h}^{T}\right) \mathbf{f}_{\mathbf{n}}
\end{array}\right.
$$

$C_{p}=\sum_{i} n_{i} c_{p i}$ is strictly positive [36] so that from equation (45), $L(T, \mathbf{n}) \rightarrow 0$ when $Z \rightarrow Z_{e}$. It is easy to show that $\mathbf{h}^{T} \mathbf{f}_{\mathbf{n}} \rightarrow 0$ and then that $\left(\tilde{w}_{1}^{-1} \tilde{w}_{2}^{T}\right) \mathbf{f}_{\mathbf{n}}$ converges to 0 proving (40).

Remark 1: Let us note that the proposition remains true as strict positivity of $C_{p}$ is always true according to the second law [36].

\section{Modification of the Lyapunov function}

The availability function combines terms that depends on temperature and mole numbers. It appears that the control efforts, especially at the beginning of the reaction, are mainly devoted to counterpart the fraction of the time derivative of the availability function which only depends on mole numbers. The most important consequence is a jacket temperature that can be excessively high or with unacceptable range for its dynamics (cf Example of Section IV) at the beginning of the reaction.

The idea is to let the part of the availability function that only depends on mole numbers $\mathbf{n}$ free by defining a new Lyapunov function. Indeed for an ideal mixture one can write (cf Proof of Lemma 1):

$$
\left(\frac{-\boldsymbol{\mu}}{T}+\frac{\boldsymbol{\mu}_{e}}{T_{e}}\right)^{T}=\boldsymbol{\Gamma}(H, \mathbf{n})+\boldsymbol{\Lambda}(\mathbf{n})
$$

Thus the availability function defined by (29) can be written in the form:

$$
\begin{aligned}
\mathcal{A}(Z) & =-\tilde{w}_{1}^{T} H-(\boldsymbol{\Gamma}(H, \mathbf{n})+\boldsymbol{\Lambda}(\mathbf{n}))^{T} \mathbf{n} \\
& =\mathcal{A}_{1}(H, \mathbf{n})+\mathcal{A}_{2}(\mathbf{n})
\end{aligned}
$$

and one can consider $\mathcal{A}_{1}$ as the new Lyapunov function. Let us first describe such decomposition in Lemma 1. Its use for control derivation is detailed in Theorem 1.

Lemma 1: In the case of an ideal mixture, the availability function (29) can be written as the sum of two positive functions $\mathcal{A}_{1}$ and $\mathcal{A}_{2}$ so that:

$$
\left\{\begin{array}{l}
\mathcal{A}_{1}(H, \mathbf{n})=-\left(\frac{1}{T}-\frac{1}{T_{e}}\right) H-\boldsymbol{\Gamma}(H, \mathbf{n})^{T} \mathbf{n} \\
\mathcal{A}_{2}(\mathbf{n})=-\boldsymbol{\Lambda}(\mathbf{n})^{T} \mathbf{n}
\end{array}\right.
$$


with

$$
\begin{aligned}
& \boldsymbol{\Gamma}(H, \mathbf{n})=\mathbf{c}_{\mathbf{p}} \ln \left(\frac{T}{T_{e}}\right)-\frac{\mathbf{h}^{T}}{T}+\frac{\mathbf{h}_{\mathbf{e}}{ }^{T}}{T_{e}} \\
& \Lambda_{k}(\mathbf{n})=R \ln \left(\frac{n_{k e} \sum_{i} n_{i}}{n_{k} \sum_{i} n_{i e}}\right)
\end{aligned}
$$

Furthermore $\mathcal{A}_{2}$ is a homogeneous function of degree one with respect to $\mathbf{n}$ and

$$
\frac{d \mathcal{A}_{1}}{d t}=-\widetilde{\boldsymbol{w}}_{\boldsymbol{r}}{ }^{T} \frac{d Z}{d t}
$$

with $\mathbf{Z}=(H, \mathbf{n}), \widetilde{\boldsymbol{w}}_{\boldsymbol{r}}{ }^{T}=\left(\widetilde{w}_{1}^{T}, \widetilde{\boldsymbol{w}}_{\boldsymbol{r} \mathbf{2}}{ }^{T}\right)=\left(\frac{1}{T}-\frac{1}{T_{e}}, \boldsymbol{\Gamma}^{T}\right)$

Proof. We first start by decomposing the chemical potential into two parts, aiming at highlighting the term which is dependent only on the species. From definition of the chemical potential:

$$
\mu_{i}=h_{i}-T s_{i}
$$

with, in the case of the ideal incompressible mixture,

$$
s_{i}=c_{p i} \ln \left(\frac{T}{T_{r e f}}\right)+s_{i r e f}-R \ln \left(\frac{n_{i}}{N}\right)
$$

with respectively $c_{p i}, s_{i r e f}$ as the heat capacity and as the reference entropy. After some basic manipulations, one can write from (52) and (53):

$$
\left(-\frac{\mu_{i}}{T}+\frac{\mu_{i e}}{T_{e}}\right)=\Gamma_{i}(H, \mathbf{n})+\Lambda_{i}(\mathbf{n})
$$

with

$$
\begin{aligned}
& \Gamma_{i}(H, \mathbf{n})=c_{p i} \ln \left(\frac{T}{T_{e}}\right)-\frac{h_{i}}{T}+\frac{h_{e i}}{T_{e}} \\
& \Lambda_{i}(\mathbf{n})=R \ln \left(\frac{n_{i e} \sum_{k} n_{k}}{n_{i} \sum_{k} n_{k e}}\right)
\end{aligned}
$$

The availability function can be written:

$$
\mathcal{A}(H, \mathbf{n})=\underbrace{-\tilde{w}_{1}^{T} H-\Gamma^{T} \mathbf{n}}_{\mathcal{A}_{1}(H, \mathbf{n})} \underbrace{-\boldsymbol{\Lambda}^{T} \mathbf{n}}_{\mathcal{A}_{2}(\mathbf{n})}
$$

$\Gamma$ and $\Lambda$ being the two column vectors of component $\Gamma_{i}$ and $\Lambda_{i}$ respectively. Furthermore firstly by using:

$$
H=\mathbf{h}^{T} \mathbf{n}
$$

it is easy to show that $\mathcal{A}_{1}=-\left(1-\frac{T}{T_{e}}+\ln \left(\frac{T}{T_{e}}\right)\right) C_{p}$. From the strict negativity of the function $f: x \rightarrow 1-x+\ln (x)$ for $x>0, x \neq 1$ and the fact that $C_{p}>0$ then $\mathcal{A}_{1}(Z)>0, \forall Z \neq Z_{e}$. Secondly, $\mathcal{A}_{2}$ is defined by:

$$
\mathcal{A}_{2}=\sum_{i}\left(R \ln \left(\frac{n_{i} \sum_{k} n_{k e}}{n_{i e} \sum_{k} n_{k}}\right)\right) n_{i}
$$


Let us note that:

$$
\frac{\partial \mathcal{A}_{2}}{\partial n_{i}}=R \ln \left(\frac{n_{i} \sum_{k} n_{k e}}{n_{i e} \sum_{k} n_{k}}\right)
$$

and that $\mathcal{A}_{2}$ is a homogeneous function of degree one with respect to $\mathbf{n}$ :

$$
\mathcal{A}_{2}=\frac{\partial \mathcal{A}_{2}^{T}}{\partial \mathbf{n}} \mathbf{n}=-\Lambda^{T} \mathbf{n}
$$

Consequently:

$$
\frac{d \mathcal{A}_{2}}{d t}=\frac{\partial \mathcal{A}_{2}}{\partial \mathbf{n}} \frac{d \mathbf{n}}{d t}=-\Lambda^{T} \frac{d \mathbf{n}}{d t}
$$

From (56):

$$
\frac{d \mathcal{A}}{d t}=-\left(\frac{1}{T}-\frac{1}{T_{e}}\right) \frac{d H}{d t}-\left(\Gamma^{T}+\Lambda^{T}\right) \frac{d \mathbf{n}}{d t}
$$

and then using (61):

$$
\frac{d \mathcal{A}_{1}}{d t}=-\widetilde{w}_{r} \frac{d Z}{d t}
$$

Let us now show the positivity of $\mathcal{A}_{2}$. $\mathcal{A}_{2}$ can be written:

$$
\mathcal{A}_{2}=\underbrace{R \sum_{i} \ln \left(\frac{n_{i}}{\sum_{k} n_{k}}\right) n_{i}}_{\Theta}-R \sum_{i} \ln \left(\frac{n_{i e}}{\sum_{k} n_{k e}}\right) n_{i}
$$

with $\Theta=R \sum_{i} \ln \left(\frac{n_{i}}{\sum_{k} n_{k}}\right) n_{i}$. One can easily show that $\Theta(\mathbf{n})$ is convex. Furthermore:

$$
\frac{\partial \Theta}{\partial n_{i}}=R \ln \left(\frac{n_{i}}{\sum_{k} n_{k}}\right) \text { and } \Theta=\frac{\partial \Theta}{\partial \mathbf{n}} \mathbf{n}
$$

Then $\mathcal{A}_{2}$ can be viewed as the distance between $\Theta(\mathbf{n})$ and its tangent plane in $\mathbf{n}_{e}$ i.e.:

$$
\mathcal{A}_{2}=\Theta(\mathbf{n})-\left(\left.\frac{\partial \Theta}{\partial \mathbf{n}}\right|_{\mathbf{n}=\mathbf{n}_{e}}\left(\mathbf{n}-\mathbf{n}_{e}\right)+\Theta\left(\mathbf{n}_{e}\right)\right)>0
$$

One can remark that $\mathcal{A}_{1}$ has very similar properties to $\mathcal{A}$ and that it can be used as a new Lyapunov function to derive stabilizing control law. It remains to concentrate the control on the thermal part of the system by avoiding the important actuator effort to control the material part (that is indirectly stabilized).

Theorem 1: The dynamic system $(27,28)$ is asymptotically stable in $Z_{e}$ if:

$$
u(Z)=-f_{H}+K_{1}(Z) \tilde{w}_{1}-\tilde{w}_{1}^{-1} \Gamma^{T} \mathbf{f}_{\mathbf{n}}
$$

with $\Gamma^{T}$ defined from (55) and with $K_{1}(Z)$ a real valued positive function. In this case $\mathcal{A}_{1}$ is a Lyapunov function for the closed loop system satisfying:

$$
\frac{d \mathcal{A}_{1}}{d t}=-K_{1}(Z) \tilde{w}_{1}^{2}<0, \forall T \neq T_{e}
$$


The function: $T \mapsto \tilde{w}_{1}^{-1} \boldsymbol{\Gamma}^{T}$ being $C^{\infty}, T$ and $u$ are $C^{\infty}$. Furthermore the closed loop temperature is monotone and does not cross $T=T_{e}$.

Proof. We have shown that $\mathcal{A}_{1}(Z)>0$ for $(Z) \neq\left(Z_{e}\right)$ and that $\mathcal{A}_{1}\left(Z_{e}\right)=0$. Consequently $\mathcal{A}_{1}$ can be considered as a new Lyapunov candidate. Replacing (67) in (51) one obtains:

$$
\frac{d \mathcal{A}_{1}}{d t}=-K_{1} \tilde{w}_{1}^{2}<0 \quad, \quad \forall T \neq T_{e}
$$

So the time derivative of $\mathcal{A}_{1}$ remains negative for $T \neq T_{e}$. From Assumption 2.1, the invariant set associated to $\dot{\mathcal{A}}_{1}=0$ at $T=T_{e}$ reduces to $Z_{e}=\left(H_{e}, \mathbf{n}_{\mathbf{e}}\right)$. Thus from LaSalle's Theorem [29] the system trajectories converge asymptotically to the equilibrium state $Z_{e}$. Once again by using (67) in the expression of the energy balance (see the proof of Proposition 2) for details), one can write:

$$
C_{p} \frac{d T}{d t}=K_{1} \tilde{w}_{1}-L(T, \mathbf{n})
$$

with

$$
L(T, \mathbf{n})=\left(\tilde{w}_{1}^{-1} \boldsymbol{\Gamma}^{T}+\boldsymbol{h}^{T}\right) \mathbf{f}_{\mathbf{n}}
$$

We can note that both the control law and the temperature derivative depend on $\tilde{w}_{1}{ }^{-1} \boldsymbol{\Gamma}^{T}=$ $\left(\mathbf{c}_{\mathbf{p}} T_{\text {ref }}-\mathbf{h}_{\text {ref }}\right)+\mathbf{c}_{\mathbf{p}}\left(\frac{1}{T}-\frac{1}{T_{e}}\right)^{-1} \ln \left(\frac{T}{T_{e}}\right)$. The function $\tilde{w}_{1}^{-1} \boldsymbol{\Gamma}^{T}$ can be derived from the function $f: x \rightarrow \ln x$ which is $C^{\infty}$ for $x>0$. Consequently by using Assumption $2.2 \tilde{w}_{1}^{-1} \boldsymbol{\Gamma}^{T} \mathbf{f}_{\mathbf{n}}$ is $C^{\infty}$. Thus the control law and the temperature profile are $C^{\infty}$. Let us now give a sketch of the proof to show that the closed loop temperature profile is strictly monotone and does not cross $T=T_{e}$. For that purpose we have to show that if $T_{0}>T_{e}$ then $\frac{d T}{d t}$ remains negative as soon as $K_{1}>0$. Reversely if $T_{0}<T_{e}$ then $\frac{d T}{d t}$ remains positive as soon as $K_{1}$ is chosen positive. Let us consider the case $T_{0}>T_{e}$. One can easily show from Equation 45 that:

$$
C_{p} \frac{d T}{d t}=K_{1} \tilde{w}_{1}-\left(\tilde{w}_{1}^{-1} \boldsymbol{\Gamma}^{T}+\boldsymbol{h}^{T}\right) \mathbf{f}_{\mathbf{n}}
$$

$C_{p}$ being positive, if $T>T_{e}$ then $\frac{d T}{d t}$ remains negative if $K_{1} \tilde{w}_{1}-\left(\tilde{w}_{1}^{-1} \boldsymbol{\Gamma}^{T}+\boldsymbol{h}^{T}\right) \mathbf{f}_{\mathbf{n}}<0$. By using the expressions of $\mathbf{f}_{\mathbf{n}}, \Gamma^{T}$, and $\boldsymbol{h}^{T}$ with respect to $T$ and $\mathbf{n}$ and the fact that the $n_{i}$ are bounded from the conservation of the volume and that the logarithm function is a concave function and the Arrhenius term is bounded on the considered domain, one can show that there exists a function $\Pi(T)$ such that:

$$
-\left(\tilde{w}_{1}^{-1} \boldsymbol{\Gamma}^{T}+\boldsymbol{h}^{T}\right) \mathbf{f}_{\mathbf{n}}<\Pi(T)
$$


and that $K_{1} \tilde{w}_{1}+\Pi(T)<0$ for all $T>T_{e}$ as soon as $K_{1}>0$. As a consequence:

$$
C_{p} \frac{d T}{d t}=K_{1} \tilde{w}_{1}-\left(\tilde{w}_{1}^{-1} \boldsymbol{\Gamma}^{T}+\boldsymbol{h}^{T}\right) \mathbf{f}_{\mathbf{n}}<K_{1} \tilde{w}_{1}+\Pi(T)<0
$$

That ends the first part of the proof. The second part of the proof can be treated with similar arguments.

Remark 2: Let us remark that

$$
\mathcal{A}=\mathcal{A}_{1}+\mathcal{A}_{2}
$$

thus

$$
\frac{d \mathcal{A}_{1}}{d t}<0 \text { is equivalent to } \frac{d \mathcal{A}}{d t}<\frac{d \mathcal{A}_{2}}{d t}
$$

As $\frac{d \mathcal{A}_{2}}{d t}$ could be positive, the use of $\mathcal{A}_{1}$ is less restrictive than the use of $\mathcal{A}$.

\section{CASE STUdy: A NON ISOTHERMAL CSTR MODEL}

The case study illustrates the efficiency of the control strategies in the case of a first-order chemical reaction: $A \rightarrow B$. In the previous section the considered control variable was $\dot{Q}$. In practice the manipulated control variable is usually the jacket temperature $T_{j}$. In this study we consider that these two variables are linked through the linear relation:

$$
\dot{Q}=\alpha\left(T_{j}-T\right)
$$

$\alpha(W / K)$ is the heat transfer coefficient. $T_{j}$ is manipulated through a Cooler/Heater supply system and has to have smooth variations. It will be shown how the design parameter $K_{1}$ can be fixed from the initial conditions and how the use of the modified Lyapunov function insures gentle variations of the control variable. In the following we consider the same assumptions as the ones made in Subsection III-A. We moreover assume that:

- The kinetics of the liquid phase reaction is given by $k_{0} e^{\frac{-E}{R T}} \frac{n_{A}}{V}, E(\mathrm{~J} / \mathrm{mol}), R(\mathrm{~J} / \mathrm{mol} / \mathrm{K})$ and $k_{0}$ are the activation energy, the gas constant and the rate constant respectively.

- The two species have the same partial molar volumes $v_{A}=v_{B}=v\left(\mathrm{~m}^{3} / \mathrm{mol}\right)$.

All the numerical data relative to this example are gathered in table I (see also [22]).

In the following we consider a regulation of the total output molar flow $F_{o}$ so that the total mass in the reactor remains constant. This guarantees the strict convexity of the availability function. 


\begin{tabular}{llll}
\hline Symb. & Numerical value & Symb. & Numerical value \\
\hline$C_{p A}$ & 75.24 & $C_{p B}$ & 60. \\
$E$ & 1046.43 & $h_{\text {Aref }}$ & 0 \\
$h_{\text {Bref }}$ & -4575 & $k_{0}$ & $0.1210^{10}$ \\
$P$ & $10^{5}$ & $P_{\text {ref }}$ & $10^{5}$ \\
$R$ & 8.314 & $T_{\text {ref }}$ & 300 \\
$\mathrm{v}$ & 0.0005 & $V$ & 0.001 \\
$\lambda$ & 0.05808 & $s_{\text {Aref }}$ & 210.4 \\
$s_{B r e f}$ & 180.2 & $\xi_{A}$ & 1 \\
$\xi_{B}$ & 1 & &
\end{tabular}

TABLE I

NUMERICAL VALUES OF PARAMETERS.

\section{A. CSTR modelling}

The balances on species $A$ and $B$ can be written:

$$
\left\{\begin{array}{l}
\frac{d n_{A}}{d t}=F_{A i}-F_{A o}-r_{\mathrm{v}} V \\
\frac{d n_{B}}{d t}=-F_{B o}+r_{\mathrm{v}} V
\end{array}\right.
$$

and the energy balance can be written:

$$
\frac{d H_{A B}}{d t}=\alpha\left(T_{j}-T\right)+F_{A i} h_{A i}-\left(F_{A o} h_{A}+F_{B o} h_{B}\right)
$$

The total volume within the reactor can be written: $V=\sum_{l=A, B} n_{l} v_{l}, v_{l}$ being the specific molar volume of component $l$. The total outlet molar flow rate $F$ is chosen so that the total volume is constant i.e. $\frac{d V}{d t}=0$ (see Subsection III-A). The outlet molar flows of species $A$ and $B$ are $F_{A o}=x_{A} F$ and $F_{B o}=x_{B} F$, with:

$$
F=\frac{v_{A} F_{A i}+\left(v_{B}-v_{A}\right) r_{v} V}{x_{A} v_{A}+x_{B} v_{B}}
$$

where $x_{A}, x_{B}, v_{A}, v_{B}$ are the molar fractions and the molar volumes of components $A$ and $B$ respectively. As a consequence, using the first balance equation in (78) and equation (79) one can write, with $k_{1}=\frac{E}{R}$ and $H_{A B}=n_{A} h_{A}+n_{B} h_{B}$ :

$$
\left\{\begin{array}{l}
\frac{d H_{A B}}{d t}=\alpha\left(T_{j}-T\right)+F_{A i}\left(h_{A i}-\beta_{1} H_{A B}\right)-\beta_{2} H_{A B} r_{V} \\
\frac{d n_{A}}{d t}=F_{A i}\left(1-\beta_{1} n_{A}\right)-\left(1+n_{A} \beta_{2}\right) r_{V} V
\end{array}\right.
$$


where $\beta_{1}=\frac{v_{A}}{x_{A} v_{A}+x_{B} v_{B}}$ and $\beta_{2}=\frac{v_{B}-v_{A}}{x_{A} v_{A}+x_{B} v_{B}} V$.

Remark 3: The system (81) can be written in the form (27) with $u=T_{j}$ by:

$$
\begin{aligned}
& f=\left(\begin{array}{c}
f_{H_{A B}} \\
f_{n_{A B}}
\end{array}\right)=\left(\begin{array}{c}
F_{A i}\left(h_{A i}-\beta_{1} H_{A B}\right)-\beta_{2} H_{A B} r_{V}-\alpha T \\
F_{A i}\left(1-\beta_{1} n_{A}\right)-\left(1+n_{A} \beta_{2}\right) r_{V} V
\end{array}\right) \\
& g=\left(\begin{array}{c}
\alpha \\
0
\end{array}\right)
\end{aligned}
$$

The steady states are computed for the following conditions (inlet molar flow of component $A$ and temperature, jacket temperature):

$$
F_{A i}=0.0183 \mathrm{~mol} \mathrm{~s}^{-1}, T_{i}=310 \mathrm{~K} \text { and } T_{j}=300 \mathrm{~K}
$$

Using these operating conditions, the system admits three steady states denoted $P_{1}, P_{2}$ and $P_{3}$. $P_{1}$ and $P_{3}$ are stable, whereas the steady state operating point $P_{2}$ is unstable. Depending on the initialization state, the chemical reaction shuts down (low temperature) toward steady state $\mathrm{P} 1$, or the reaction runs out of control (high temperature) toward the steady state P3. It can be illustrated by loop simulations (Fig. 3), with respect to the initial conditions $\left(C 1: T_{0}=\right.$ $\left.340 \mathrm{~K}, n_{A 0}=0.6 \mathrm{~mol}\right),\left(C 2: T_{0}=325 \mathrm{~K}, n_{A 0}=1.8 \mathrm{~mol}\right),\left(C 3: T_{0}=300 \mathrm{~K}, n_{A 0}=1.6 \mathrm{~mol}\right)$ and $\left(C 4: T_{0}=300 \mathrm{~K}, n_{A 0}=0.6 \mathrm{~mol}\right)$.

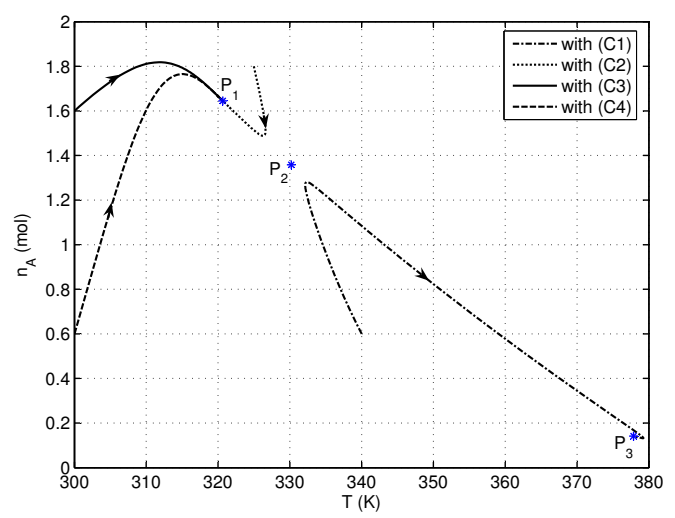

Fig. 3. The representation of the open loop phase plane.

\section{B. Controller synthesis and simulations}

This section illustrates the performances of the different control strategies of Section III. The objective is to stabilize the system around the unstable point $P_{2}$, i.e. $P_{2}\left(Z_{e}\right)$. First, we consider 
the exponential stabilization using the feedback law (33) with initial state $\left(T_{0}=300 \mathrm{~K}, n_{A 0}=\right.$ $1 \mathrm{~mol}) . K_{1}=0.035$ is computed from the initial condition and in such a way that $\dot{Q}(t=0)=0$.
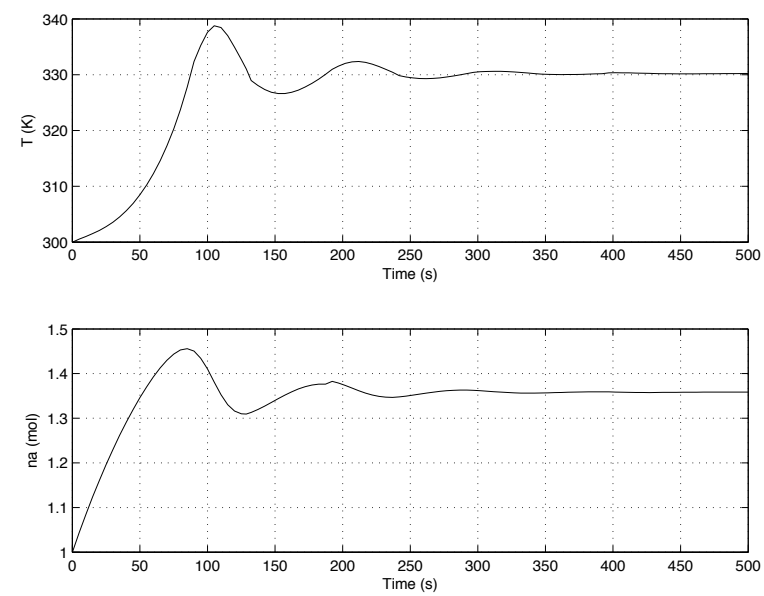

Fig. 4. Temperature and composition responses.

Figure 4 shows that the system is stable around $P_{2}$ and that the feedback plays its role. One can also notice that $T$ has an overshoot and crosses many time $T_{e}=300 \mathrm{~K}$. The response of the composition has the same behavior.
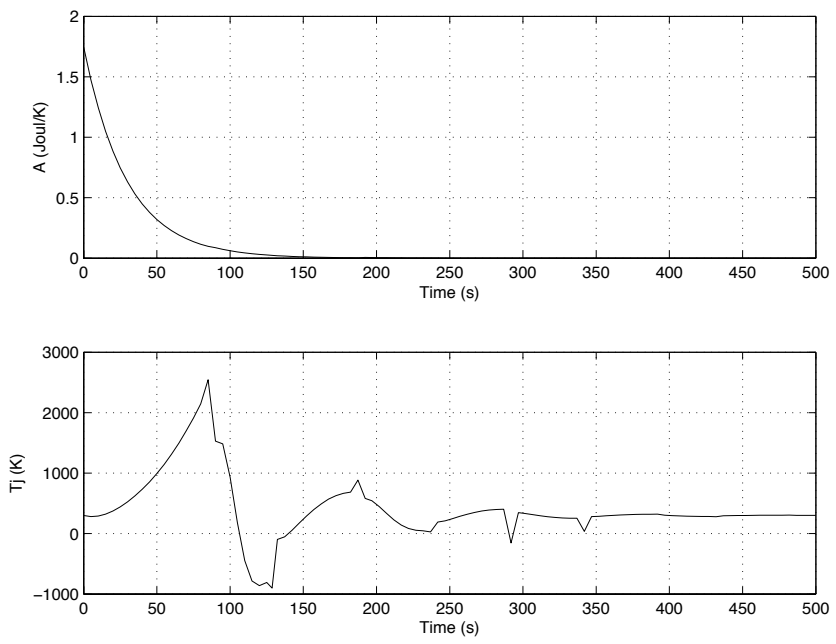

Fig. 5. Availability function and jacket temperature.

In Figure 5 we see that the availability function decreases exponentially as it is imposed by the 
control strategy. However, due to the inversion of $\tilde{w}_{1}$ and the temperature profile in Figure 4 and the control variable, i.e. the jacket temperature, presents many singularities with inadmissible values.

Let us now consider the control strategy (38). Figure 6 shows that again the system is stable around $P_{2}$.
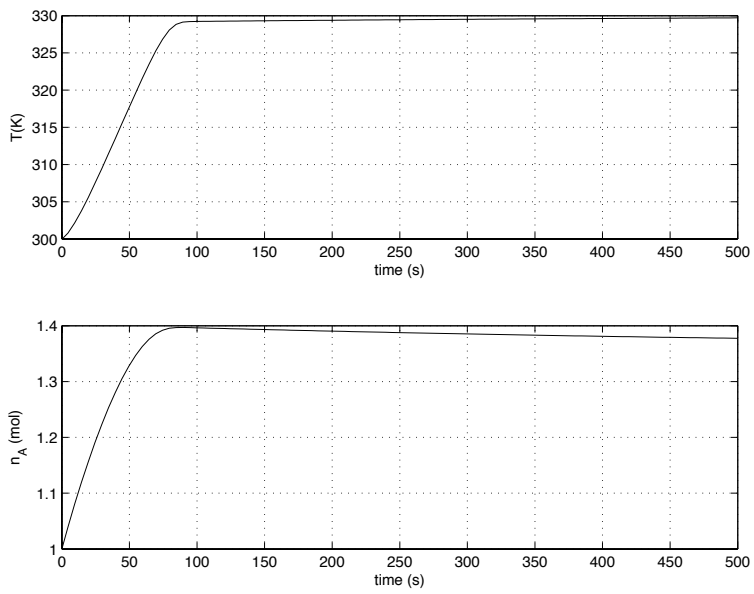

Fig. 6. Temperature and composition responses.

Even if the control variable remains bounded, as it is illustrated in Figure 7, the jacket temperature is out of physical bounds. Indeed the jacket temperature reaches $1200 \mathrm{~K}$.

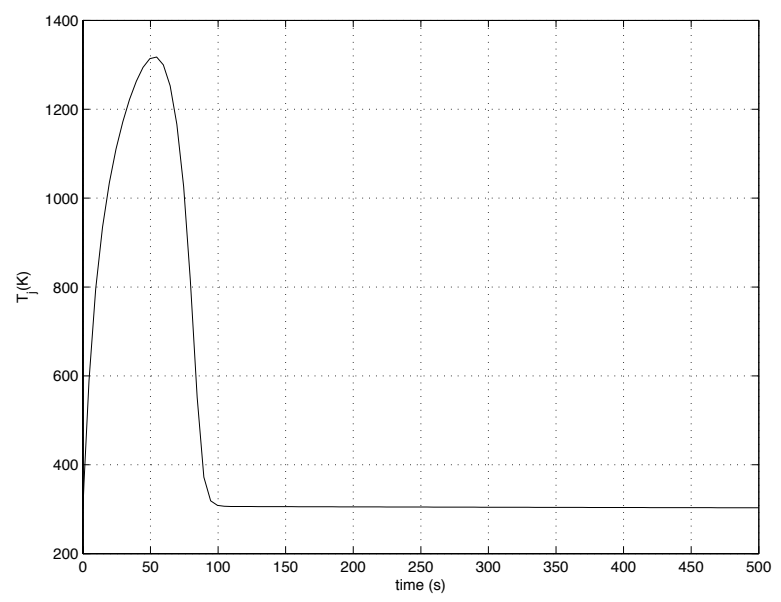

Fig. 7. Jacket temperature. 
To overcome this drawback one can use Theorem 1 that leads in the case of this example to Corollary 1.

Corollary 1: The system (81) with the non linear feedback law (with fixed $T_{i}$ and $F_{A i}$ )

$$
T_{j}=\frac{1}{\alpha}\left(K_{1} \widetilde{w}_{1}-f_{H_{A B}}-\Gamma_{A B} \widetilde{w}_{1}^{-1} \frac{d n_{A}}{d t}\right)
$$

where:

$$
\left\{\begin{array}{l}
\widetilde{w}_{1}=\left(\frac{1}{T}-\frac{1}{T_{e}}\right) \\
f_{H_{A B}}=F_{A i}\left(h_{A i}-\beta_{1} H_{A B}\right)-\beta_{2} H_{A B} r_{V}-\alpha T \\
\Gamma_{A B}=\left(\left(c_{p A}-\frac{\beta_{1}}{\beta_{1}+\beta_{2}} c_{p B}\right) T_{r e f}-\left(h_{A r e f}-\frac{\beta_{1}}{\beta_{1}+\beta_{2}} h_{B r e f}\right)\right) \\
\quad\left(\frac{1}{T}-\frac{1}{T_{e}}\right)+\left(c_{p A}-\frac{\beta_{1}}{\beta_{1}+\beta_{2}} c_{p B}\right) \ln \left(\frac{T}{T_{e}}\right)
\end{array}\right.
$$

is stable and asymptotically converges to the desired operating point $P_{2}$. Furthermore the closed loop behavior of the temperature is given by:

$$
C_{p} \frac{d T}{d t}=K_{1}\left(\frac{1}{T}-\frac{1}{T_{e}}\right)+L(T) \frac{d n_{A}}{d t}
$$

where

$$
L(T)=\left(-\Gamma_{A B}\left(\frac{1}{T}-\frac{1}{T_{e}}\right)^{-1}-\left(h_{A}-\frac{\beta_{1}}{\beta_{1}+\beta_{2}} h_{B}\right)\right)
$$

Proof. Equation (84) is deduced from equation (67) taking into account that $\dot{Q}=\alpha\left(T_{j}-T\right)$ and $n_{A} v_{A}+n_{B} v_{B}=$ const. Indeed $\mathbf{f}_{\mathbf{n}}=\left(\frac{d n_{A}}{d t}, \quad-\frac{\beta_{1}}{\beta_{1}+\beta_{2}} \frac{d n_{A}}{d t}\right)^{T}$ and $-\frac{\boldsymbol{\mu}^{T}}{T}+\frac{\boldsymbol{\mu}_{e}^{T}}{T_{e}}=\left(-\frac{\mu_{A}}{T}+\frac{\mu_{A e}}{T_{e}}, \quad-\frac{\mu_{B}^{T}}{T}+\frac{\mu_{B e}^{T}}{T_{e}}\right)$ leading to (84).

Remark 4: The considered control variable is the jacket temperature $T_{j}$. Practically in order to avoid the initial jump of the control variable $T_{j}$, the parameter $K_{1}$ can be tuned such that $\dot{Q}=0$ at $t=0$. In the closed loop case such constraint restricts the domain of the initial conditions compatible with $K_{1}>0$. To determine this domain of validity one has to find the initial conditions compatible with (84). This is equivalent to

$$
\left[K_{1} \widetilde{w}_{1}-f_{H_{A B}}-\Gamma_{A B} \widetilde{w}_{1}^{-1} \frac{d n_{A}}{d t}\right]_{t=0}=0
$$

with $K_{1}>0$. In our simple case this domain (union of domain 1 and domain 2) is delimitated by the function $\Xi(T)$ as depicted in Fig. 8. In the general case such domain has to be numerically solved.

In what follows we consider that the state variables are measured (if not, one has to implement an observer as proposed in [12]). The system is closed using the state feedback law derived using 


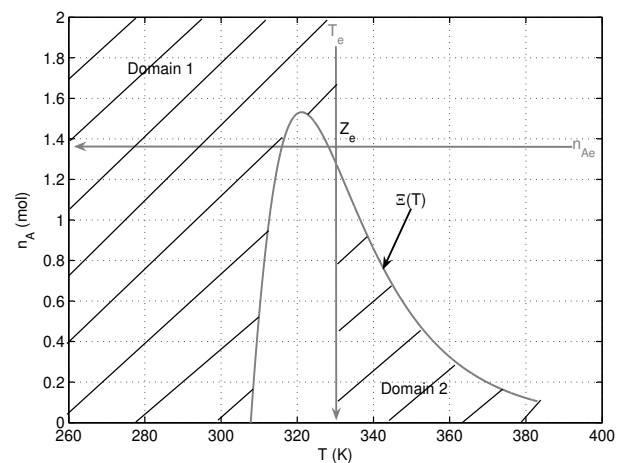

Fig. 8. Domain of validity of initial conditions

$\mathcal{A}_{1}$ as the Lyapunov function. In Figure 9 the closed loop phase plane is represented. We can see that for all the considered initial conditions the system converges to the desired operating point $\mathrm{P} 2$. Let us consider the particular case (C3): at the beginning of the reaction $n_{A}$ is increasing; it means that the reaction is consuming less $n_{A}$ than the quantity supplied to the reactor. As a consequence the control law tends to impose high jacket temperature to initiate the reaction and to stabilize it asymptotically to P2. Figure 10 shows that the control variable $T_{j}$ is admissible in

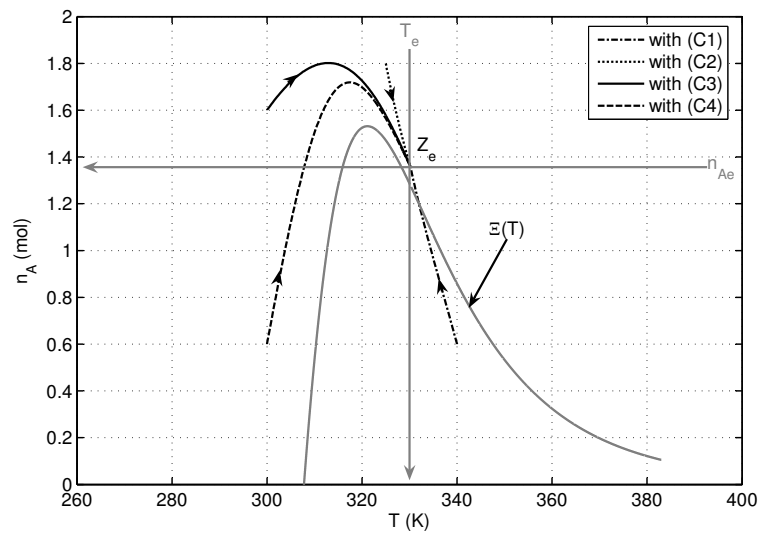

Fig. 9. Representation of the closed loop phase plane.

terms of amplitude and dynamics. This interesting property is linked to the fact that the system is not constrained too much as it was the case in [22]. Let us now examine the evolution of $\mathcal{A}_{1}, \mathcal{A}_{2}$ and $\mathcal{A}$ in Figure 11, Figure 12, Figure 13 respectively. In Figure 11 we can see that 


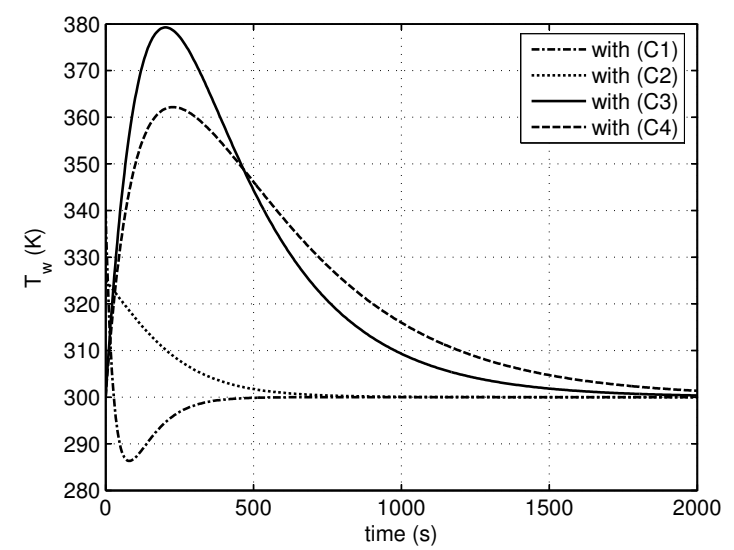

Fig. 10. The feedback law $T_{j}$

$\mathcal{A}_{1}$ decreases with time. It is in accordance with the control strategy insuring that $\frac{d \mathcal{A}_{1}}{d t}<0$. Figure 12 shows that $\mathcal{A}_{2}$ does not always decrease. Indeed $\mathcal{A}_{2}$ increases for the cases $(C 3)$ and
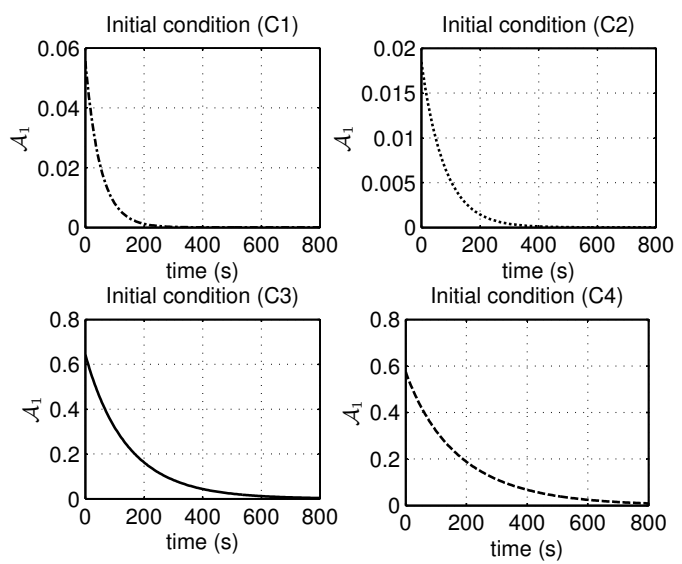

Fig. 11. Time evolution of $\mathcal{A}_{1}$.

(C4). Furthermore its growth rate is greater than the rate of decrease of $\mathcal{A}_{1}$. As a consequence one can note from Figure 13 that the associate function $\mathcal{A}$ admits positive variations in these two cases. If $\mathcal{A}$ would have been chosen as the Lyapunov function, the control variable would have to counterpart these positive variations of $\mathcal{A}_{2}$ involving significant control values. It is in accordance with the results of Figure 7 corresponding to the use of $\mathcal{A}$ as a Lyapunov function.

The results presented here are satisfactory from both a qualitative and a quantitative point 

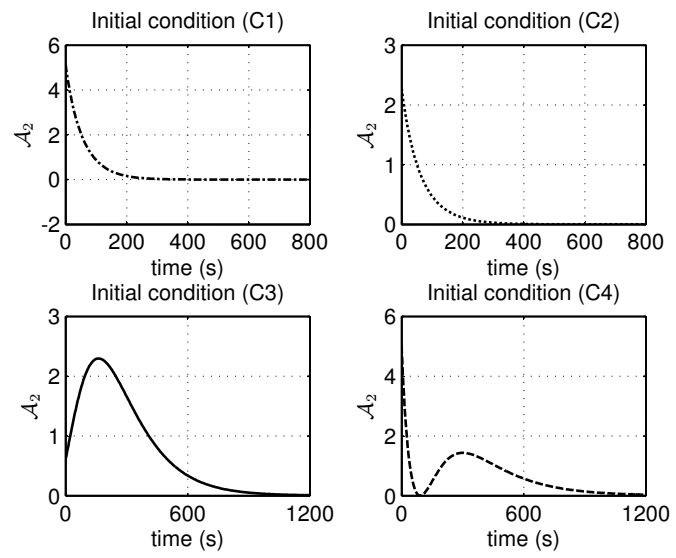

Fig. 12. Time evolution of $\mathcal{A}_{2}$.

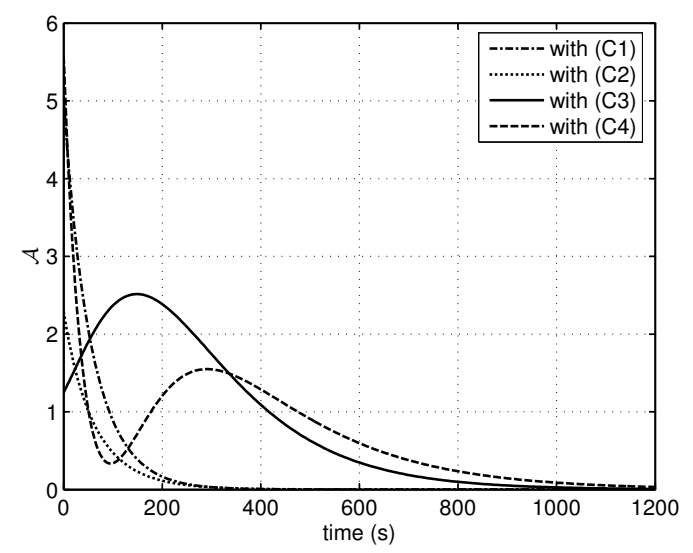

Fig. 13. Time evolution of $\mathcal{A}$.

of view. We showed that thermodynamics naturally proposes a Lyapunov function usable for dynamic control. Nevertheless, in the proposed control strategy, the closed loop dynamics is imposed by the initial conditions through the tuning parameter $K_{1}$. This is the reason why we are now looking for dynamic controllers with additional degrees of freedom.

\section{Conclusions}

The second law of thermodynamics allows to define the so called Availability function that is usable for control purposes when one of the extensive variables is fixed. In this paper we consider a perfect regulation of volume to satisfy this constraint. If no attention is paid to the 
admissibility of the control variable, the global asymptotic stabilizing control law as well as the exponential stabilizing control law can easily be derived.

When bounded control variable is looked for it is possible to insure asymptotic stability. Nevertheless in practice such a strategy can lead to unbounded control or control variables with strong variations. A modified strategy considers the thermal part of the Availability function as a closed loop Lyapunov function. These different strategies have been applied to a first order chemical reaction. The relaxation of the Lyapunov function has proven to be efficient since it gives bounded control variables. This work shows that the energetic exchanges within the system is important. A natural extension of this work uses the port Hamiltonian formulation for modeling and control. The first result of such an approach can be found in [24].

\section{REFERENCES}

[1] Alonso, A.A. and Ydstie, B.E. (1996). Process systems, passivity and the second law of thermodynamics, Computers \& Chemical Engineering, 20(2):1119-1124.

[2] Alonso, A.A. and Ydstie, B.E. (2001). Stabilization of Distributed Systems using Irreversible Thermodynamics, Automatica, 37:1739-1755.

[3] Alvarez-Ramirez, J. and Femat, R. (1999). Robust PI stabilization of a class of chemical reactors. Systems \& Control Letters, 38(4-5):219-225.

[4] Antelo, L.T. and Otero-Muras, I. and Banga, J. R. and Alonso, A. A. (2007). A Systematic Approach to Plant-Wide Control Based On Thermodynamics. Computers \& Chemical Engineering,31(5-6):677-691.

[5] Antonelli, R. and Astolfi, A. (2003). Continuous stirred tank reactors: easy to stabilise? Automatica, 39:1817-1827.

[6] Aris, R. and Amundson, N.R. (1973). Mathematical Methods in Chemical Engineering Vol.II, Prentice-Hall.

[7] Biagiola, S.I. and Figueroa, J.L. (2004). A high gain nonlinear observer: application to the control of an unstable nonlinear process. Computers and Chemical Engineering, 28(9):1881-1898.

[8] Brogliato, B. and Lozano, R. and Maschke, B. and Egeland, O. (2007). Dissipative systems analysis and control. Springer, London, 2nd edition. 
[9] Callen, H.B. (1985). Thermodynamics and an introduction to thermostatics. JohnWiley \& Sons Inc, 2nd ed. New York.

[10] Costa, V.A.F. (2008). Volume balance equation, availability analysis and the fundamental relation in the thermodynamics for the heat transfer man. International Journal of Heat and Mass Transfer, 51(11-12):2871-2876.

[11] Couenne, F. and Jallut, C. and Maschke, B. and Breedveld, P.C. and Tayakout, M. (2006). Bond graph modelling for chemical reactors. Mathematical and Computer Modelling of Dynamical Systems, 12(2-3):1-9.

[12] Dochain, D. and Couenne, F. and Jallut, C. (2009). Enthalpy Based Modelling and Design of Asymptotic Observers for Chemical Reactors. International Journal of Control, Volume 82, Issue 8 August 2009 , pages 1389 - 1403

[13] Döfler, F. and Johnsen, J.K. and Allgöwer, F. (2009). An introduction to interconnection and damping assignment passivity-based control in process engineering. Journal of Process Control, 19, 1413-1426.

[14] Eberard, D. and Maschke, B. and van der Schaft, A. (2007). An extension of pseudoHamiltonian systems to the thermodynamic space: towards a geometry of non-equilibrium thermodynamics. Reports on Mathematical Physics, 60(2), 175-198.

[15] Favache, A. and Dochain, D. (2009a). Power-shaping control of an exothermic continuous stirred tank reactor (CSTR). ADCHEM, Turkey. 101-110.

[16] Favache, A. and Dochain, D. (2009b). Thermodynamics and chemical systems stability: The CSTR case study revisited, Journal of Process Control, 19(3), 2009. pp. 371-379

[17] Gavalas, G (1968). Nonlinear Differential equations of chemical reacting systems. SpringerVerlag.

[18] Glansdorff, P. and Prigogine, I (1971). Thermodynamic theory of structure, stability and fluctuations. Wiley-Interscience.

[19] Guo, B. and Jiang, A. and Hua, X. and Jutan, A. (2001). Nonlinear adaptive control for multivariable chemical processes, Chemical Engineering Science, 56, 6781-6791.

[20] Hangos, K. M. and Alonso, A. A. and Perkins, J. D. and Ydstie, B. E. (1999). Thermodynamic approach to the structural stability of process plants, AIChE journal,1 vol. 45, no4, pp. $802-816$

[21] Hangos, K. M. and Bokor, J. and Szederkenyi, G. (2001). Hamiltonian view on process 
systems, AIChE journal,vol. 47, no 8, pp. 1819-1831.

[22] Hoang, H. and Couenne, F. and Jallut, C. and Le Gorrec, Y. (2008). Lyapunov based control for non isothermal continuous stirred tank reactor. Proceedings of the 17th World Congress of the IFAC, July 6-11, 2008, Seoul, Korea.

[23] Hoang, H. and Couenne, F. and Le Gorrec, Y. (2009). Thermodynamic approach for Lyapunov based control. Proceeding of International Symposium on Advanced Control of Chemical Processes ADCHEM, July 12-15, Istanbul, Turkey, Pages 367-372.

[24] Hoang, H. and Couenne, F. and Jallut, C and Le Gorrec, Y. (2010). Port Hamiltonian based modeling and control of exothermic Continuous Stirred Tank Reactors. IFAC Workshop NOLCOS, Bologna.

[25] Hoang, $\mathrm{H}$ and Couenne, F and Jallut, C and Le Gorrec, Y. (2011). The port Hamiltonian approach to modeling and control of Continuous Stirred Tank Reactors, Journal of Process Control, Vol. 21, issue 10, December, 1449-1458

[26] Hua, X. and Jutan, A. (2000). Nonlinear Inferential Cascade Control of Exothermic Fixedbed Reactors. AICHE Journal, 46:980-996.

[27] Jana, A.K. and Samanta, A.N. and Ganguly, S. (2005). Globally linearized control on diabatic continuous stirred tank reactor: a case study. ISA Transactions, 44:423-444.

[28] Jillson, R.J. and Ydstie, B.E. (2007). Process networks with decentralized inventory and flow control,Journal of Process Control, Volume 17, Issue 5, June 2007, Pages 399-413

[29] Khallil, H.K. (2002). Nonlinear systems. $3^{\text {rd }}$ edition, Prentice Hall, ISBN: 0131227408.

[30] Keenan, J.H. (1951). Avaibility and irreversibility in thermodynamics. british Journal of Applied Physics, 2:183-192.

[31] Luyben, W.L. (1990). Process Modeling, Simulation, and Control for Chemical Engineers.McGraw-Hill, Singapore, 1990.

[32] Ramirez, H. and Sbarbaro, D. and Ortega, R. (2009). On the control of non-linear processes: An IDAPBC approach. Journal of Process Control, 19(3):405-414

[33] Read, N.K. and Ray, W.H. (1998). Application of nonlinear dynamic analysis to the identification and control of nonlinear systems -III. n-Dimensional systems. Journal of Process Control, 8(1):35-46.

[34] Rothfuss, R. and Rudolph, J. and Zeitz, M. (1996). Flatness based control of a nonlinear chemical reactor. Automatica, 32(10):1433-1439. 
[35] Ruszkowski, M. and Garcia-Osorio, V. and Ydstie, B.E. (2005). Passivity based control of transport reaction systems. AIChE Journal, 51:3147-3166.

[36] Sandlers, SI (1999). Chemical and Engineering Thermodynamics. Wiley and Sons, 3rd edition.

[37] van der Schaft, A. (2000a). Port-controlled Hamiltonian systems: towards a theory for control and design of nonlinear physical systems. SICE journal, 39(2), 91-98.

[38] van der Schaft, A. (2000b). $L_{2}$-gain and passivity techniques in nonlinear control. SpringerVerlag, London, 2nd edt.

[39] Viel, F. and Jadot, F. and Bastin, G. (1997a). Global stabilization of exothermic chemical reactors under input constraints. Automatica, 33(8):1437-1448.

[40] Viel, F. and Jadot, F. and Bastin, G. (1997b). Robust feedback stabilization of chemical reactors. IEEE Transactions on Automatic Control, 42(4):473-481.

[41] Willems, J. (1970). Stability Theory of Dynamical Systems. Nelson, New York.

[42] Ydstie, B.E. and Alonso, A.A. (1997). Process systems and passivity via the ClausiusPlanck inequality. Systems Control Letters, 30(5):253-264. 